\title{
CALCULATION OF A NON-REFLECTIVE CONNECTION IN A COAXIAL LINE
}

\author{
V.M. Morozov, V.I. Magro* \\ Oles Honchar Dnipro National University, Dnipro, Ukraine \\ *e-mail:magrov@i.ua
}

The calculation of the non-reflective connection in the coaxial line is performed by the integral equation method. The connection of coaxial lines with a significant difference in geometric dimensions is considered. A system of equations is obtained that allows calculating the reflection coefficient of the $T$-wave from such an inhomogeneity. This technique makes it possible to calculate a multistage coaxial waveguide in order to minimize the reflection coefficient from inhomogeneities. coupling.

Keywords: multistage coaxial transition, coaxial waveguide, integral equation, non-reflective waveguide

Received 04.06.2021; Received in revised form 12.07.2021; Accepted 15.08.2021

\section{Introduction}

Discontinuous changes in the cross section of the coaxial line are considered in [1,2]. The transitions between the coaxial line and the waveguide are also considered [3, 4]. A change in the transverse dimensions of the lines to be connected is accompanied by a change in the structure of the electromagnetic field and the wave impedance of such lines. If the transverse dimensions of the lines to be connected differ by more than five times, then it is necessary to build a multistage transition. An example of the construction of such a transition can be as shown in Fig. 1. In addition to the multistage transition, a cone-shaped transition can be used (Fig. 2) [2]. A cone-shaped transition has constant characteristic impedance along its entire length only if both cones have a common vertex. In a cone-shaped transition, the field is distorted at the junction of the cylindrical segments with the conical segments. The disadvantages of a tapered transition include a long transition length.

When calculating a multi-step transition, it is impossible to apply the standard equivalent diagrams that are used to calculate transitions with a slight difference in the transverse dimensions of the connected lines. Here the method of computer electrodynamics [3] or the exact classical electrodynamics calculation [5] can be applied.

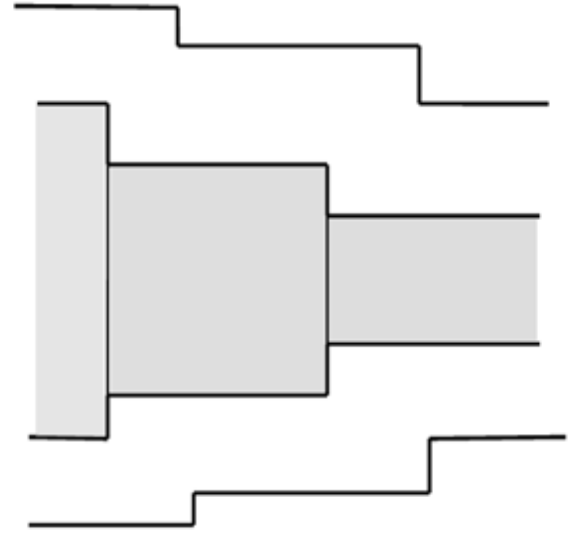

Fig.1. Multistage coaxial transition

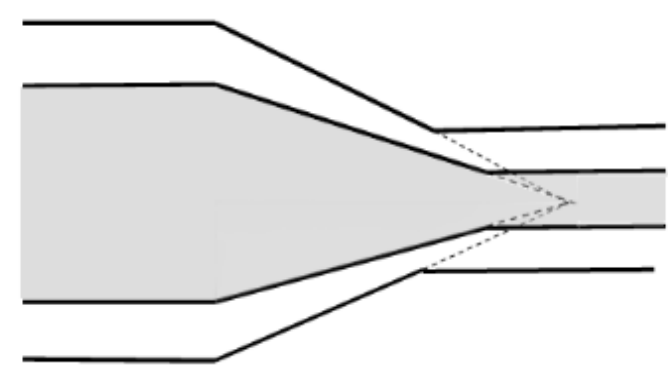

Fig. 2. Cone-shaped transition

\section{Formulation of the problem}

In fig. 3 shows a cross-section of a single stepped junction in a coaxial waveguide. Let us conditionally divide the region of determining the field in the coaxial waveguide into three regions. Region A: $0 \leq z \leq l, \quad r_{0}<\rho<r_{3}$. Region B: $\infty \leq z<0, \quad r_{0}<\rho<r_{3}$. 
Region C: $l \leq z<+\infty, \quad r_{0}<\rho<r_{2}$ (fig. 3).

In region $\mathrm{B}$, the main $\mathrm{T}$-wave is excited at a point $z=-\infty$. The walls of a stepped coaxial transition are assumed to be ideally conducting, and the medium in the line is homogeneous and isotropic. We assume that in region $\mathrm{C}$ the line is loaded with a matched load.

Let us write the equation for the field components for each area. In region A:

$$
\begin{gathered}
E_{r A}=\frac{1}{r}\left(A_{0}^{\prime} e^{-j k z}+A_{0}^{\prime \prime} e^{j k z}\right)+\sum_{m=1}^{\infty}\left(A_{m}^{+} e^{-\gamma_{A m} z}+A_{m}^{-} e^{\gamma_{A m} z}\right) Z_{1}\left(\chi_{A m} r\right), \\
H_{\varphi A}=\frac{1}{\eta r}\left(A_{0}^{\prime} e^{-j k z}-A_{0}^{\prime \prime} e^{j k z}\right)+\sum_{m=1}^{\infty} Y_{A m}^{+}\left(A_{m}^{+} e^{-\gamma_{A m} z}+A_{m}^{-} e^{\gamma_{A m} z}\right) Z_{1}\left(\chi_{A m} r\right),
\end{gathered}
$$

here $\eta=\sqrt{\mu / \varepsilon}$. In region $\mathrm{B}$ :

$$
\begin{gathered}
E_{r B}=\frac{1}{r}\left(B_{0}^{\prime} e^{-j k z}+B_{0}^{\prime \prime} e^{j k z}\right)+\sum_{m=1}^{\infty} B_{m}^{-} e^{\gamma_{B m} z} Z_{1}\left(\chi_{B m} r\right), \\
H_{\varphi B}=\frac{1}{\eta r}\left(B_{0}^{\prime} e^{-j k z}-B_{0}^{\prime \prime} e^{j k z}\right)+\sum_{m=1}^{\infty} Y_{B m}^{-} B_{m}^{-} e^{\gamma_{B m} z} Z_{1}\left(\chi_{B m} r\right) .
\end{gathered}
$$

In region $\mathrm{C}$ :

$$
\begin{gathered}
E_{r C}=\frac{1}{r}\left(C_{0}^{\prime} e^{-j k z}+C_{0}^{\prime \prime} e^{j k z}\right)+\sum_{m=1}^{\infty} C_{m}^{+} e^{-\gamma_{C m} z} Z_{1}\left(\chi_{C m} r\right), \\
H_{\varphi C}=\frac{1}{\eta r}\left(C_{0}^{\prime} e^{-j k z}-C_{0}^{\prime \prime} e^{j k z}\right)+\sum_{m=1}^{\infty} Y_{C m}^{+} C_{m}^{+} e^{-\gamma_{C m} z} Z_{1}\left(\chi_{C m} r\right) .
\end{gathered}
$$

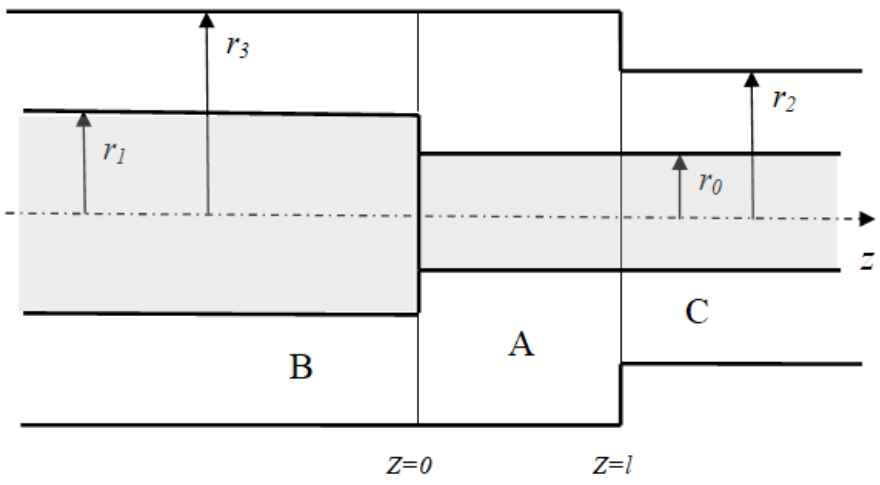

Fig.3. Single stepped junction in a coaxial waveguide 
All quantities that enter into the equation for region A are determined from the following relations:

$$
\begin{gathered}
Z_{n}\left(\chi_{A m} r\right)=J_{n}\left(\chi_{A m} r\right)+G_{A m}\left(\chi_{A m} r\right) \\
G_{A m}\left(\chi_{A m} r\right)=-\frac{J_{0}\left(\chi_{A m} r_{0}\right)}{N_{0}\left(\chi_{A m} r_{0}\right)}=-\frac{J_{0}\left(\chi_{A m} r_{3}\right)}{N_{0}\left(\chi_{A m} r_{3}\right)} \\
Y_{A m}^{+}=\frac{j \omega \varepsilon}{\gamma_{A m}}, \quad \gamma_{A m}=\sqrt{\chi_{A m}^{2}-k^{2}} .
\end{gathered}
$$

Here $\chi_{A m}$ is determined from the equation:

$$
J_{0}\left(\chi_{A m} r_{0}\right) N_{0}\left(\chi_{A m} r_{3}\right)-J_{0}\left(\chi_{A m} r_{3}\right) N_{0}\left(\chi_{A m} r_{0}\right)=0 .
$$

\section{Non-reflective connection calculation}

Let us write down the equations for the regions $\mathrm{A}$ and $\mathrm{B}$ at the point $z=0$; for regions $\mathrm{A}$ and $\mathrm{C}$ at the point $z=l$. The conditions for "stitching" the fields at the boundaries of the division of the regions are as follows: in point $z=0$ $E_{r B}=E_{r A}, \quad r_{1}<r<r_{3} ; E_{r A}=0, \quad r_{0}<r<r_{1} ; H_{\varphi B}=H_{\varphi A}, \quad r_{1}<r<r_{3} ;$ in point $z=l$ $E_{r B}=E_{r A}, \quad r_{1}<r<r_{3} ; E_{r A}=0, \quad r_{2}<r<r_{3} ; H_{\varphi B}=H_{\varphi A}, \quad r_{1}<r<r_{3}$.

Using the conditions for "matching" electromagnetic fields, we obtain six equations.

$$
\begin{gathered}
\frac{1}{r}\left(A_{0}^{\prime}+A_{0}^{\prime \prime}\right)+\sum_{m=1}^{\infty}\left(A_{m}^{+}+A_{m}^{-}\right) Z_{1}\left(\chi_{A m} r\right)=\frac{1}{r}\left(B_{0}^{\prime}+B_{0}^{\prime \prime}\right)+\sum_{m=1}^{\infty} B_{m}^{-} e^{\gamma_{B m} z} Z_{1}\left(\chi_{B m} r\right), \quad r_{1} \\
\mathrm{r}_{1}<\mathrm{r}<\mathrm{r}_{3}
\end{gathered}
$$




$$
\begin{gathered}
\frac{1}{r}\left(A_{0}^{\prime} e^{-j k l}+A_{0}^{\prime \prime} e^{j k l}\right)+\sum_{m=1}^{\infty}\left(A_{m}^{+} e^{-\gamma_{A m} l}+A_{m}^{-} e^{\gamma_{A m} l}\right) Z_{1}\left(\chi_{A m} r\right)=0, \quad r_{2}<r^{<}<r_{3} \\
\frac{1}{\eta r}\left(A_{0}^{\prime} e^{-j k l}-A_{0}^{\prime \prime} e^{j k l}\right)+\sum_{m=1}^{\infty} Y_{A m}^{+}\left(A_{m}^{+} e^{-\gamma_{A m} l}+A_{m}^{-} e^{\gamma_{A m} l}\right) Z_{1}\left(\chi_{A m} r\right)= \\
=\frac{1}{\eta r} C_{0}^{\prime} e^{-j k l}+\sum_{m=1}^{\infty} Y_{C m}^{+} C_{m}^{+} e^{-\gamma_{C m} l} Z_{1}\left(\chi_{C m} r\right), \quad r_{0}<r<r_{2}
\end{gathered}
$$

We will integrate over $r$ in the range from $r_{1}$ to $r_{3}$ in area B and from $r_{0}$ to $r_{3}$ in area A. From equations (1) and (2) we obtain

$$
\left(A_{0}^{\prime}+A_{0}^{\prime \prime}\right) \ln \left(\frac{r_{3}}{r_{0}}\right)-\left(B_{0}^{\prime}+B_{0}^{\prime \prime}\right) \ln \left(\frac{r_{3}}{r_{1}}\right)=0 ;
$$

from equations (4) and (5) we obtain

$$
\left(A_{0}^{\prime} e^{-j k l}+A_{0}^{\prime \prime} e^{j k l}\right) \ln \left(\frac{r_{3}}{r_{0}}\right)-C_{0}^{\prime} e^{-j k l} \ln \left(\frac{r_{2}}{r_{0}}\right)=0 ;
$$

from equation (3) we obtain

$$
\frac{1}{\eta}\left(A_{0}^{\prime}-A_{0}^{\prime \prime}\right) \ln \left(\frac{r_{3}}{r_{1}}\right)-\left(B_{0}^{\prime}+B_{0}^{\prime \prime}\right) \ln \left(\frac{r_{3}}{r_{1}}\right)+\sum_{m=1}^{\infty} Y_{A m}^{+}\left(A_{m}^{+}-A_{m}^{-}\right) \frac{Z_{1}\left(\chi_{A m} r_{1}\right)}{\chi_{A m}}=0
$$

from equation (6) we obtain

$$
\frac{1}{\eta}\left(A_{0}^{\prime} e^{-j k l}-A_{0}^{\prime \prime} e^{j k l}\right) \ln \left(\frac{r_{2}}{r_{0}}\right)-C_{0}^{\prime} e^{-j k l} \ln \left(\frac{r_{2}}{r_{0}}\right)-\sum_{m=1}^{\infty} Y_{A m}^{+}\left(A_{m}^{+} e^{-\gamma_{A m} l}+A_{m}^{-} e^{\gamma_{A m} l}\right) \frac{Z_{0}\left(\chi_{A m} r_{2}\right)}{\chi_{A m}}=0
$$

We use the property of orthogonality of eigenfunctions. We multiply expressions (1) and (2) by $r Z_{1}\left(\chi_{A m} r\right)$, then add the left and right sides of the obtained equalities, and then integrate from $r_{1}$ to $r_{3}$ in the section $z=-0$. In the section $z=+0$, integration can be extended from $r_{0}$ to $r_{3}$, then we get

$$
\begin{aligned}
\left(A_{0}^{\prime}\right. & \left.+A_{0}^{\prime \prime}\right) \int_{r_{0}}^{r_{3}} Z_{1}\left(\chi_{A n} r\right) d r+\sum_{m=1}^{\infty}\left(A_{m}^{+}+A_{m}^{-}\right) \int_{r_{0}}^{r_{3}} Z_{1}\left(\chi_{A m} r\right) Z_{1}\left(\chi_{A n} r\right) r d r= \\
= & \int_{r_{1}}^{r_{3}}\left(B_{0}^{\prime}+B_{0}^{\prime \prime}\right) Z_{1}\left(\chi_{A n} r\right) d r+\sum_{m=1}^{\infty} B_{m}^{-} \int_{r_{1}}^{r_{3}} Z_{1}\left(\chi_{B m} r\right) Z_{1}\left(\chi_{A n} r\right) r d r .
\end{aligned}
$$


We perform similar actions for expressions (4) and (5). We multiply both sides of expression (3) by $r Z_{1}\left(\chi_{B n} r\right)$ and integrate from $r_{1}$ to $r_{3}$. We multiply both sides of expression (6) by $r Z_{1}\left(\chi_{C n} r\right)$ and integrate from $r_{0}$ to $r_{2}$. Next, we use the known transformations from [6]:

$$
\begin{gathered}
\int_{r_{0}}^{r_{3}} \frac{Z_{1}\left(\chi_{A n} r\right)}{\chi_{A n}} d\left(r \chi_{A n}\right)=0 ; \\
\int_{r_{0}}^{r_{3}} Z_{1}\left(\chi_{A m} r\right) Z_{1}\left(\chi_{A n} r\right) r d r=0 ; \\
\int_{r_{1}}^{r_{3}} Z_{1}\left(\chi_{B m} r\right) Z_{1}\left(\chi_{A n} r\right) r d r=\frac{\chi_{A n} r_{1} Z_{1}\left(\chi_{B m} r_{1}\right) Z_{0}\left(\chi_{A n} r_{1}\right)}{\chi_{A n}^{2}-\chi_{B m}^{2}} ; \\
\int_{r_{0}}^{r_{3}} Z_{1}^{2}\left(\chi_{A n} r\right) d r=\frac{1}{2}\left[r_{3}^{2} Z_{1}^{2}\left(\chi_{A n} r_{3}\right)-r_{0}^{2} Z_{1}^{2}\left(\chi_{A n} r_{0}\right)\right]=\frac{\varphi\left(\chi_{A n}, r_{3}, r_{0}\right)}{2 \chi_{A n}^{2}} .
\end{gathered}
$$

Then from the last expression we get that

$$
\varphi\left(\chi_{A n}, r_{3}, r_{0}\right)=\left[\chi_{A n} r_{3} Z_{1}\left(\chi_{A n} r_{3}\right)\right]^{2}-\left[\chi_{A n} r_{0} Z_{1}\left(\chi_{A n} r_{0}\right)\right]^{2} .
$$

Using the transformations discussed above, the following expressions can be obtained:

$$
\begin{gathered}
\left(A_{0}^{\prime}+A_{0}^{\prime \prime}\right) \frac{\varphi\left(\chi_{A n}, r_{3}, r_{0}\right)}{2 \chi_{A n}^{2}}-\left(B_{0}^{\prime}+B_{0}^{\prime \prime}\right) \frac{Z_{1}\left(\chi_{A n} r_{1}\right)}{\chi_{A n}}-\sum_{m=1}^{\infty} B_{m} \frac{\chi_{A n} r_{1} Z_{1}\left(\chi_{B m} r_{1}\right) Z_{0}\left(\chi_{A n} r_{1}\right)}{\chi_{A n}^{2}-\chi_{B m}^{2}}=0 \\
\left(A_{m}^{+} e^{-\gamma_{A m} z}+A_{m}^{-} e^{\gamma_{A m} z}\right) \frac{\varphi\left(\chi_{A n}, r_{3}, r_{0}\right)}{2 \chi_{A n}^{2}}-C_{0}^{\prime} e^{-j k l} \frac{Z_{0}\left(\chi_{A n} r_{2}\right)}{\chi_{A n}}+ \\
+\sum_{m=1}^{\infty} C_{m}^{+} e^{-\gamma_{C m} z} \frac{\chi_{A n} r_{2} Z_{1}\left(\chi_{C m} r_{2}\right) Z_{1}\left(\chi_{A n} r_{2}\right)}{\chi_{A n}^{2}-\chi_{C m}^{2}}=0 \\
Y_{B n}^{-} \frac{\varphi\left(\chi_{B n}, r_{3}, r_{1}\right)}{2 \chi_{B n}^{2}}+\sum_{m=1}^{\infty} Y_{A m}^{+}\left(A_{m}^{+}-A_{m}^{-}\right) \frac{\chi_{A n} r_{1} Z_{1}\left(\chi_{B n} r_{1}\right) Z_{1}\left(\chi_{A m} r_{1}\right)}{\chi_{B n}^{2}-\chi_{A m}^{2}}=0 \\
Y_{C n}^{+} C_{n}^{+} e^{-\gamma_{C n} l} \frac{\varphi\left(\chi_{C n}, r_{2}, r_{0}\right)}{2 \chi_{C n}^{2}}-\sum_{m=1}^{\infty} Y_{A m}^{+}\left(A_{m}^{+} e^{-\gamma_{A m} l}-A_{m}^{-} e^{\gamma_{A m} l}\right) I_{m n}=0
\end{gathered}
$$


here

$$
\begin{gathered}
I_{m n}=\int_{r_{0}}^{r_{2}} Z_{1}\left(\chi_{A m} r\right) Z_{1}\left(\chi_{C n} r\right) r d r=\frac{-\chi_{A m} r_{2} Z_{0}\left(\chi_{A m} r_{2}\right) Z_{1}\left(\chi_{C n} r_{2}\right)}{\chi_{A m}^{2}-\chi_{C n}^{2}} ; \\
\varphi\left(\chi_{C n}, r_{2}, r_{0}\right)=\left[\chi_{C n} r_{2} Z_{1}\left(\chi_{C n} r_{2}\right)\right]^{2}-\left[\chi_{C n} r_{0} Z_{1}\left(\chi_{C n} r_{0}\right)\right]^{2} .
\end{gathered}
$$

Expressions (7) - (10) and (12) - (15) represent an infinite homogeneous system of algebraic equations. For a system to have a nonzero solution, the determinant of this system must be equal to zero. When calculating, it is necessary to restrict oneself to a finite number of higher types of wave's $m$. The number $m$ is chosen based on the required calculation accuracy. The reflection coefficient from inhomogeneity the T-wave inhomogeneity is determined by the relation $\Gamma=B_{0}^{\prime \prime} / B_{0}^{\prime}$.

\section{Conclusions}

The paper proposes an accurate electrodynamics calculation of a non-reflective connection of coaxial waveguides. The proposed technique makes it possible to calculate a multistage coaxial transition for the case of a significant difference in the transverse dimensions of the connected coaxial waveguides. Taking into account the fact that the inhomogeneities caused by the change in the cross-section of the line are not interacting, you can choose the minimum value of the geometric dimension 1 . This makes it possible to significantly reduce the longitudinal length of the multistage transition in comparison with the tapered transition and to reduce the mass and size characteristics of the transition.

\section{References}

1. Morozov, V.M. Application of the method of integral equation for calculating a steptransition in a coaxial waveguide / V.M. Morozov, V.I. Magro // Journal of Physics and Electronics. - 2020. - Vol. 28, No. 1. - P. 69-76.

2. Meinke H.H. Taschenbuch der hochfrequenztechnik / H.H. Meinke, F.W. Gundlach. - Springer, 1992. - $576 \mathrm{p}$.

3. Yu, K.W. The analysis of a coaxial-to-waveguide transition using FDTD with cylindrical to rectangular cell interpolation scheme / Kyung-Wan Yu, Sung-Choon Kang, Hee-Jin Kang, Jae-Hoon Choi, Jin-Dae Kim // ETRI Journal. - 1999. - Vol. 21. - No. 2. - P. 1-8.

4. Stepanenko, P.Ya. Koaksial'nyy stupenchatyy perekhod mezhdu pryamougol'nym i koaksial'nym volnovodami /P.Ya. Stepanenko // Izvestiya vysshikh uchebnykh zavedeniy. Radioelektronika. - 1993. - Vol. 36. - No. 6. - P. 15-26.

5. Gnilenko, A.B. Method of overlapping regions for solving electromagnetic problems / A.B. Gnilenko, V.I. Magro // Prociding of XXII ${ }^{\text {nd }}$ International seminar/workshop on direct and inverse problems of electromagnetic and acoustic wave theory (DIPED-2017). - 2017. - Dnipro, Ukraine - P.36-41.

6. Handbook of mathematical functions with formulas, graph and mathematical tables / edited by M. Abramovvits, I.A. Stegun. - Courier Corporation, 1965. - 1046 p. 